\title{
Clinical utility gene card for: Cystinosis
}

\author{
Elena Levtchenko ${ }^{\star, 1}$, Lambertus van den Heuvel $^{1,2}$, Francesco Emma ${ }^{3}$ and Corinne Antignac ${ }^{4,5}$ \\ European Journal of Human Genetics (2014) 22, doi:10.1038/ejhg.2013.204; published online 18 September 2013
}

\section{DISEASE CHARACTERISTICS \\ 1.1 Name of the disease (synonyms) \\ Cystinosis.}

\subsection{OMIM\# of the disease}

Nephropathic infantile form (MIM \#219800), nephropathic juvenile form (MIM \#219900) and non-nephropathic adult form (MIM \#219750).

1.3 Name of the analysed genes or DNA/chromosome segments CTNS, 17p13.2.

\subsection{OMIM\# of the gene(s)}

606272 .

\subsection{Mutational spectrum}

Multi-exon 57-kb deletion, small insertions, deletions, duplications, point mutations (missense, nonsense), splice-site mutations, promoter mutations, genomic rearrangements. ${ }^{1-6}$

The $57-\mathrm{kb}$ deletion is detected in up to $76 \%$ of affected northern European alleles and is due to a founder effect arising around the middle of the first millennium AD., ${ }^{1,2}$

Currently, approximately 100 mutations in the CTNS are described by HGMD (http://www.hgmd.cf.ac.uk/ac/index.php), however, novel mutations are still being reported, especially when genetically different populations are tested. ${ }^{7,8}$

The standard reference sequence indicating reported variants (ENSG00000040531) and a reference for exon numbering (ENST00000046640) can be found at www.ensembl.org.

\subsection{Analytical methods}

The 57-kb deletion: rapid PCR assay with the 57-kb deletion breakpoint primer sets, FISH analysis, and MLPA typing. Sanger sequencing of the total coding region, the exon-intron boundaries, and promoter region of the CTNS gene. Analysis of microsatellite markers for detection of uniparental heterodisomy of the chromosome 17.6

\subsection{Analytical validation}

The segregation of the mutations should be confirmed in the parents. Pathogenicity of novel missense variants has to be verified by testing a set of at least 100 control chromosomes of the same ethnic origin and by in-silico prediction methods (like SIFT, PolyPhen and/or Align). CTNS gene transcripts should be analysed in patients with exon-skipping or splice-site mutations. ${ }^{9}$ Analysis of functional consequences of the CTNS mutation on protein level in cell models is available in a few laboratories in the world.

1.8 Estimated frequency of the disease (Incidence at birth ('birth prevalence') or population prevalence. If known to be variable between ethnic groups, please report)

Birth prevalence: 1:100 000 to 1:200 000. Increased incidence in some populations: for example Brittany, France: 1:26000.

1.9 If applicable, prevalence in the ethnic group of the investigated person

Not applicable.

\subsection{Diagnostic setting}

\begin{tabular}{lll}
\hline & Yes & No \\
A. (Differential) diagnostics & $\Downarrow$ & $\square$ \\
B. Predictive Testing & $\Downarrow$ & $\square$ \\
C. Risk assessment in relatives & $\Downarrow$ & $\square$ \\
D. Prenatal & $\Downarrow$ & $\square$
\end{tabular}

\section{Comment:}

The common 57-kb deletion encompasses the first nine exons and a part of exon 10 of the CTNS gene and the upstream $5^{\prime}$ region that encodes for the CARKL gene and the first two non-coding alternative exons of the transient receptor potential vanilloid 1 receptor (TRPV1) gene.

So far, there is no evidence confirming pathological involvement of the CARKL and the TRPV1 genes in the clinical phenotype of cystinosis patients.

The CARKL gene encodes for the enzyme sedoheptulose kinase responsible for the phosphorylation of sedoheptulose into sedoheptulose-7-phosphate within the pentose phosphate pathway. Patients carrying the common 57-kb deletion accumulate sedoheptulose. Increased concentration of sedoheptulose in blood or urine can be used for identifying patients with the homozygous $57-\mathrm{kb}$ deletion. ${ }^{10,11}$

The TRPV1 gene encodes for the TRPV1 expressed in the primary sensory neurons and activated by heat and various chemical compounds, including capsaicin. ${ }^{12}$ Two first non-coding exons of the TRPV1 are removed by the $57-\mathrm{kb}$ deletion. Hypothetically, the reduced sweating and crafting for spicy food described in cystinosis patients might be attributed to a decreased function of the TRPPV1 receptor.

\footnotetext{
${ }^{1}$ Department of Pediatric Nephrology \& Growth and Regeneration, University Hospitals Leuven, Katholieke Universiteit Leuven, Leuven, Belgium; ${ }^{2}$ Department of Pediatric Nephrology, Radboud University Nijmegen, Nijmegen, The Netherlands; ${ }^{3}$ Division of Nephrology, Bambino Gesù Children's Hospital, IRCCS, Rome, Italy; ${ }^{4}$ Inserm U983, Institut Imagine, Université Paris Descartes, Paris, France; ${ }^{5}$ Department of Genetics, Assistance Publique, Hôpitaux de Paris, Necker Hospital, Paris, France

${ }^{*}$ Correspondence: Professor E Levtchenko, Department of Pediatric Nephrology \& Growth and Regeneration, University Hospitals Leuven, Katholieke Universiteit Leuven, Herestraat 49, 3000 Leuven, Belgium. Tel: + 3216 343822; Fax: + 3216 343842; E-mail: elena.levtchenko@uzleuven.be
} 


\section{TEST CHARACTERISTICS}

\begin{tabular}{|c|c|c|c|c|}
\hline & \multicolumn{2}{|c|}{ Genotype or disease } & \multirow{2}{*}{$\begin{array}{l}\text { A: True positives } \\
\text { B: False positives }\end{array}$} & \multirow{2}{*}{$\begin{array}{l}\text { C: False negative } \\
\text { D: True negative }\end{array}$} \\
\hline & Present & Absent & & \\
\hline \multicolumn{5}{|l|}{ Test } \\
\hline \multirow[t]{2}{*}{ Positive } & $A$ & B & Sensitivity: & $A /(A+C)$ \\
\hline & & & Specificity: & $D /(D+B)$ \\
\hline \multirow[t]{2}{*}{ Negative } & C & D & Positive predictive value: & $A /(A+B)$ \\
\hline & & & Negative predictive value: & $D /(C+D)$ \\
\hline
\end{tabular}

\subsection{Analytical sensitivity}

(proportion of positive tests if the genotype is present)

Close to $100 \%$.

Not all found variants have been tested functionally, ${ }^{13}$ and therefore a theoretical possibility exists that some of them might be rare polymorphisms. The pathogenicity of most mutants has been confirmed by in-silico prediction methods.

\subsection{Analytical specificity}

(proportion of negative tests if the genotype is not present)

Close to $100 \%$.

\subsection{Clinical sensitivity}

\section{(proportion of positive tests if the disease is present)}

The clinical sensitivity can be dependent on variable factors, such as age or family history. In such cases a general statement should be given, even if a quantification can only be made case by case.

Close to $100 \%$.

\subsection{Clinical specificity}

(proportion of negative tests if the disease is not present)

The clinical specificity can be dependent on variable factors, such as age or family history. In such cases a general statement should be given, even if a quantification can only be made case by case.

Close to $100 \%$.

\subsection{Positive clinical predictive value}

(life-time risk to develop the disease if the test is positive) $100 \%$.

\subsection{Negative clinical predictive value}

(Probability of not developing the disease if the test is negative) Assume an increased risk based on family history for a non-affected person. Allelic and locus heterogeneity may need to be considered.

Index case in that family had been tested:

$100 \%$.

Index case in that family had not been tested:

Depending on the degree of relationship and consanguinity $(25 \%$ risk for developing disease in siblings). Less than $1 \%$ chance to develop the disease in other relatives in populations with a low degree of consanguinity.

\section{CLINICAL UTILITY}

3.1 (Differential) diagnostics: The tested person is clinically affected

(To be answered if in 1.9 'A' was marked)

\subsubsection{Can a diagnosis be made other than through a genetic test?}

\begin{tabular}{llc}
\hline No & $\square$ (continue with 3.1.4) \\
Yes & $\square$ \\
& Clinically & \\
Imaging & $\otimes^{*}$ \\
Endoscopy & $\square$ \\
Biochemistry & $\square$ \\
Electrophysiology & $\square$ \\
Other (please describe) & Split-lamp cornea examination
\end{tabular}

*Although not widely available, in vivo confocal microscopy of the cornea or skin are valuable tools for diagnosing cystinosis. ${ }^{14,15}$

\subsubsection{Describe the burden of alternative diagnostic methods to the} patient

In the majority of cases, the diagnosis of cystinosis is based on the high degree of clinical suspicion (presence of renal Fanconi syndrome, corneal cystine accumulation) and on the biochemical detection of elevated cystine levels in white blood cells (WBC) or preferentially in polymorphonuclear cells having a higher degree of cystine accumulation.

Biochemical methods of cystine measurement (high-performance liquid chromatography or tandem mass spectrometry) are time consuming, require rapid blood processing for cell isolation and can only be performed in specialised laboratories experienced in this measurement.

Young children may have rather low cystine accumulation in WBC and low amount of corneal cystine crystals, complicating the diagnosis of cystinosis shortly after birth and during the first months of life.

Corneal crystals may be absent until 16 months of age. ${ }^{16}$

3.1.3 How is the cost effectiveness of alternative diagnostic methods to be judged?

The cost effectiveness of clinical eye investigation and biochemical cystine measurements is very high.

The differences in price between tests depend on the laboratory and the country where testing is performed. Clinical eye examination is always cheaper compared with biochemical or genetic testing, but it is not contributive in patients below 1-2 years of age. ${ }^{16}$

3.1.4 Will disease management be influenced by the result of a genetic test?

No $\square$

Yes $\otimes$

Therapy Early genetic diagnosis of cystinosis allows early initiation

(please describe) of treatment with cystine-lowering drug cysteamine ( $\beta$-mercaptoethylamine).

Prognosis Renal and extra-renal function survival in cystinosis

(please describe) is dependent on the age of onset of cysteamine therapy. ${ }^{16-21}$

Management (1) Symptomatic treatment of cystinosis consists of free

(please describe) access to water and replacement therapy with potassium, bicarbonate, phosphate salts and vitamin D, and in older patients with thyroxin, insulin and sex hormones (if required). Young patients are frequently treated with indomethacin to reduce diuresis and urinary electrolyte losses. Growth hormone may be indicated in patients with stunted growth despite adequate nutrition and electrolyte balance. ${ }^{20-22}$ 
(2) Systemic cysteamine therapy $\left(1.3-1.9 \mathrm{~g} / \mathrm{m}^{2} /\right.$ day) should be administered immediately after making the diagnosis and continued life-long (also after kidney transplantation) to reduce cystine WBC levels to $<1 \mathrm{nmol}$ cystine/mg protein. ${ }^{20,22}$

(3) Topical cysteamine eye drops $(0.5 \%)$ should be administered at least 4 times daily to reduce corneal cystine crystals. ${ }^{16}$

(4) Renal transplantation

3.2 Predictive Setting: The tested person is clinically unaffected but carries an increased risk based on family history

(To be answered if in 1.9 'B' was marked)

\subsubsection{Will the result of a genetic test influence lifestyle and prevention?}

If the test result is positive (please describe):

In affected persons cysteamine therapy should be started immediately after the diagnosis. Early therapy postpones renal failure and the development of extra-renal manifestations of cystinosis.

If the test result is negative (please describe)

No further medical follow-up will be required in subjects from families with a known genetic defect. In case of an unknown mutation in the family, at-risk individuals should be followed medically.

3.2.2 Which options in view of lifestyle and prevention does a person at risk have if no genetic test has been done (please describe)?

No life-style adaptations or other type of prevention can be applied. No medical follow-up will be required in persons with a negative genetic test within a family with identified mutations.

3.3 Genetic risk assessment in family members of a diseased person (To be answered if in 1.9 'C' was marked)

\subsubsection{Does the result of a genetic test resolve the genetic situation in that family? \\ Yes.}

3.3.2 Can a genetic test in the index patient save genetic or other tests in family members?

Yes. If the index case has known mutations, siblings, parents and other family members can be screened for disease or carriership.

3.3.3 Does a positive genetic test result in the index patient enable a predictive test in a family member?

Yes. Genetic test results in the index case allow prenatal diagnosis in the next siblings. In populations with a high degree of consanguinity, carriership in partners can be tested as well.

\subsection{Prenatal diagnosis}

(To be answered if in 1.9 ' $\mathrm{D}$ ' was marked)

\subsubsection{Does a positive genetic test result in the index patient enable a prenatal diagnosis?}

Yes.

\section{IF APPLICABLE, FURTHER CONSEQUENCES OF TESTING}

Please assume that the result of a genetic test has no immediate medical consequences. Is there any evidence that a genetic test is nevertheless useful for the patient or his/her relatives? (Please describe)

Yes, for performing genetic counselling in a family.

In general, there is no consensus on genetic testing of partners of affected female patients planning pregnancies. Some genetic laboratories recommend to screen partners for the mutations common in their population (for example: for 57-kb deletion in the Northern European population). Male cystinosis patients are infertile. ${ }^{23}$

\section{CONFLICT OF INTEREST}

The authors declare no conflict of interest.

\section{ACKNOWLEDGEMENTS}

This work was supported by EuroGentest2 (Unit 2: 'Genetic testing as part of health care'), a Coordination Action under FP7 (Grant Agreement Number 261469) and the European Society of Human Genetics. E Levtchenko is supported by the Fund for Scientific Research, Flanders (Belgium) (FWO Vlaanderen) (Grant Agreement 1801110N). E Levtchenko, L van den Heuvel, F Emma and C Antignac are supported by the Cystinosis Research Foundation, USA.

1 Town M, Jean G, Cherqui S et al: A novel gene encoding an integral membrane protein is mutated in nephropathic cystinosis. Nat Genet 1998; 18: 319-324.

2 Forestier L, Jean G, Attard M et al: Molecular characterization of CTNS deletions in nephropathic cystinosis: development of a PCR-based detection assay. Am J Hum Genet 1999; 65: 353-359.

3 Kalatzis V, Cohen-Solal L, Cordier B et al: Identification of 14 novel CTNS mutations and characterization of seven splice site mutations associated with cystinosis. Hum Mutat 2002; 20: 439-446.

4 Mason S, Pepe G, Dall'Amico R et al: Mutational spectrum of the CTNS gene in Italy. Eur J Hum Genet 2003; 11: 503-508.

5 Phornphutkul C, Anikster Y, Huizing $\mathrm{M}$ et al: The promoter of a lysosomal membrane transporter gene, CTNS, binds $\mathrm{Sp}-1$, shares sequences with the promoter of an adjacent gene, CARKL, and causes cystinosis if mutated in a critical region. Am J Hum Genet 2001; 69: 712-721.

6 Lebre AS, Morinière V, Dunand O, Bensman A, Morichon-Delvallez N, Antignac C: Maternal uniparental heterodisomy of chromosome 17 in a patient with nephropathic cystinosis. Eur J Hum Genet 2009; 17: 1019-1023.

7 Topaloglu R, Vilboux T, Coskun T et al: Genetic basis of cystinosis in Turkish patients: a single-center experience. Pediatr Nephrol 2012; 27: 115-121.

8 Shahkarami S, Galehdari H, Ahmadzadeh A et al: The first molecular genetics analysis of individuals suffering from nephropatic cystinosis in the Southwestern Iran. Nefrologia 2013; 33: 308-315

9 Taranta A, Wilmer MJ, van den Heuvel LP et al: Analysis of CTNS gene transcripts in nephropathic cystinosis. Pediatr Nephrol 2010; 25: 1263-1267.

10 Wamelink MM, Struys EA, Jansen EE et al: Sedoheptulokinase deficiency due to a 57-kb deletion in cystinosis patients causes urinary accumulation of sedoheptulose: elucidation of the CARKL gene. Hum Mutat 2008; 29: 532-536.

11 Wamelink MM, Struys EA, Jansen EE et al: Elevated concentrations of sedoheptulose in bloodspots of patients with cystinosis caused by the 57-kb deletion: implications for diagnostics and neonatal screening. Mol Genet Metab 2011; 102: 339-342.

12 Gees M, Owsianik G, Nilius B, Voets TTRP: Channels. Compr Physiol 2012; 2: 563-608.

13 Kalatzis V, Nevo N, Cherqui S, Gasnier B, Antignac C: Molecular pathogenesis of cystinosis: effect of CTNS mutations on the transport activity and subcellular localization of cystinosin. Hum Mol Genet 2004; 13: 1361-1371.

14 Labbé A, Niaudet P, Loirat C, Charbit M, Guest G, Baudouin C: In vivo confocal microscopy and anterior segment optical coherence tomography analysis of the cornea in nephropathic cystinosis. Ophthalmology 2009. 116. 870-876.

15 Chiavérini C, Kang HY, Sillard L et al: In vivo reflectance confocal microscopy of the skin: a noninvasive means of assessing body cystine accumulation in infantile cystinosis. J Am Acad Dermatol 2013; 68: e111-e116.

16 Gahl WA, Kuehl EM, Iwata F, Lindblad A, Kaiser-Kupfer MI: Corneal crystals in nephropathic cystinosis: natural history and treatment with cysteamine eyedrops. Mol Genet Metab 2000; 71: 100-120.

17 Markello TC, Bernardini IM, Gahl WA: Improved renal function in children with cystinosis treated with cysteamine. N Engl J Med 1993; 328: 1157-1162.

18 Greco M, Brugnara M, Zaffanello M, Taranta A, Pastore A, Emma F: Long-term outcome of nephropathic cystinosis: a 20-year single-center experience. Pediatr Nephrol 2010; 25: 2459-2467.

19 Nesterova G, Gahl W: Nephropathic cystinosis: late complications of a multisystemic disease. Pediatr Nephrol 2008; 23: 863-878.

20 Kleta R, Kaskel F, Dohil R et al: First NIH/Office of Rare Diseases Conference on Cystinosis: past, present, and future. Pediatr Nephrol 2005; 20: 452-454.

21 Van Stralen KJ, Emma F, Jager KJ et al: Improvement in the renal prognosis in nephropathic cystinosis. Clin J Am Soc Nephrol 2011; 6: 2485-2491.

22 Wilmer MJ, Schoeber JP, van den Heuvel LP, Levtchenko EN: Cystinosis: practical tools for diagnosis and treatment. Pediatr Nephrol 2011; 26: 205-215.

23 Besouw MT, Kremer JA, Janssen MC, Levtchenko EN: Fertility status in male cystinosis patients treated with cysteamine. Fertil Steril 2010; 93: 1880-1883. 